\title{
AVALIAÇÃO DO QUADRIL DE AMPUTADOS TRANSFEMORAL DURANTE CONTRAÇÃO ISOMÉTRICA EM DINAMÔMETRO ISOCINÉTICO
}

\author{
HIP EVALUATION OF TRANSFEMORAL AMPUTEES DURING ISOMETRIC CONTRACTION \\ IN ISOKINETIC DYNAMOMETER
}

\section{EVALUACIÓN DE LA CADERA DE AMPUTADOS TRANSFEMORALES DURANTE CONTRACCIÓN ISOMÉTRICA EN DINAMÓMETRO ISOCINÉTICO}

\author{
Vera Regina Fernandes da Silva \\ Marães' (Fisioterapeuta) \\ Bruna Oliveira de Almeida Marques \\ da Cruz' (Discente de Fisioterapia) \\ Juliana Aparecida Moreira' \\ (Discente de Fisioterapia) \\ Tainara Ferreira de Sampaio \\ (Discente de Fisioterapia) \\ Camila Cadena Almeida ${ }^{1}$ \\ (Fisioterapeuta) \\ Patrícia Azevedo Garcia \\ (Fisioterapeuta)
}

1. Faculdade de Ceilândia, Universidade de Brasília, Brasília, DF, Brasil.

\section{Correspondência:}

SQN 206 Bloco B Apt 105. Brasília, Asa Norte. Distrito Federal, Brasil. 70844020.

veraregina@unb.br; vrmaraes@gmail.com

\section{RESUMO}

Introdução: A amputação transfemoral leva a uma série de alterações funcionais na biomecânica corporal que podem interferir no cotidiano do amputado, podendo gerar, ainda, padrões de postura e de marcha para compensar a perda do membro. Objetivo: Avaliar e comparar as respostas musculares do quadril de amputados transfemorais durante contrações isométricas, utilizando-se um dinamômetro isocinético. Método: Participaram do estudo quatro voluntários, sendo dois amputados e dois não amputados. Foi realizada avaliação isométrica no dinamômetro isocinético nas angulações de 30 e 60 graus com duração de 10 segundos. Cada série tinha um total de seis repetições, contabilizando três flexões e três extensões e um intervalo de 20 segundos para cada repetição. Resultados: 0 membro amputado apresenta menor pico de torque em relação ao não amputado, tanto na flexão como extensão do quadril a 30 e a 60 graus. Conclusão: Devido à amputação, os indivíduos apresentam menor força muscular de quadril, fato que pode prejudicar as atividades diárias como caminhar, sentar e levantar, e subir e descer escadas.

Palavras-chave: amputação, dinamômetro de força muscular, resistência física.

\section{ABSTRACT}

Introduction: Transfemoral amputation leads to a series of functional changes in the body biomechanics that can interfere with the daily life of the amputee, generating patterns of posture and gait to compensate for the loss of the limb. Objective: This study aims to evaluate and compare the muscular responses of the hip during isometric contractions in transfemoral amputees, using an isokinetic dynamometer. Method: The study included four volunteers, two amputees and two non-amputees. Evaluation was performed using an isokinetic dynamometer in isometric angles of 30 and 60 degrees of 10 seconds duration. Each series was made of six repetitions, composed by three pushups and three extensions with a 20 seconds interval between each sequence. Results: The amputated limb showed a lower peak torque compared to the non-amputee, both in flexion and in extension of the hip at 30 and 60 degrees. Conclusion: Due to amputation, individuals have less hip muscle strength, which may impair daily activities such as walking, sitting and standing, climbing and descending stairs.

Keywords: amputation, muscle strength dynamometer, physical endurance.

\section{RESUMO}

Introducción: La amputación transfemoral lleva a una serie de alteraciones funcionales en la biomecánica corporal que pueden interferir en el cotidiano del amputado pudiendo generar, además, estándares de postura y de marcha para compensar la pérdida del miembro. Objetivo: Evaluar y comparar las respuestas musculares de la cadera de amputados transfemorales durante contracciones isométricas, utilizándose un dinamómetro isocinético. Método: Participaron en el estudio cuatro voluntarios, siendo dos amputados y dos no amputados. Fue realizada evaluación isométrica en el dinamómetro isocinético en las angulaciones de 30 y 60 grados con duración de 10 segundos. Cada serie tenía un total de seis repeticiones, contabilizando tres flexiones y tres extensiones y un intervalo de 20 segundos para cada repetición. Resultados: El miembro amputado presenta menor pico de torque en relación al no amputado, tanto en la flexión como extensión de la cadera a 30 y a 60 grados. Conclusión: Debido a la amputación, los individuos presentan menor fuerza muscular de cadera, hecho que puede perjudicar las actividades diarias como caminar, sentarse y levantarse, subir y bajar escaleras.

Palabras clave: amputación, dinamómetro de fuerza muscular, resistencia física. 


\section{INTRODUÇÃO}

O termo amputação pode ser definido como sendo a retirada, geralmente cirúrgica, total ou parcial de um membro'. A amputação transfemoral refere-se à retirada de parte do membro inferior entre a articulação do joelho e a do quadril. Este procedimento leva a uma série de alterações funcionais na biomecânica corporal que podem interferir no cotidiano do amputado gerando, ainda, padrões de postura e de marcha para compensar a perda do membro.

O desempenho muscular é a capacidade de trabalho de um músculo. Força muscular é um termo amplo que se refere à habilidade do tecido contrátil de produzir tensão e uma força resultante com base nas demandas impostas sobre o músculo². A força muscular é um importante componente da prestação desportiva, por essa razão a avaliação da força muscular com recurso da dinamometria isocinética é largamente utilizada ${ }^{3}$.

Os valores de força, além de ter um componente individual e genético, são também afetados pelo sexo, idade, nível do atleta e pela modalidade esportiva praticada 4 . Os amputados transfemorais apresentam significativa redução da força no membro amputado comparado ao não amputado 5 .

A contração isométrica ou contração estática é definida quando o músculo desenvolve tensão sem sofrer encurtamento, ou seja, o músculo desenvolve tensão, mas não há alteração em seu comprimento externo ou no ângulo da articular ${ }^{2}$. O dinamômetro isocinético tem sido usado amplamente como o método para avaliação de força muscular. Sua característica principal é a velocidade angular constante e a resistência dependente do esforço realizado.

As mensurações são confiáveis, seguras, precisas, objetivas e reprodutíveis. As desvantagens estão ainda no preço elevado. Outro aspecto está no fato de que o aparelho isocinético não realiza o gesto ou o movimento específico de uma determinada modalidade esportiva. Portanto, o esforço realizado não envolve a energia cinética nas várias articulações, e sim em uma única articulação, estando o restante do corpo sem deslocamento. Os movimentos são realizados normalmente sob a forma de exercício de cadeia cinética aberta6.

Durante a avaliação no isocinético, fatores como ambiente, a capacitação do examinador, as condições do aparelho e o estímulo ao qual o paciente é submetido interferem nos resultados. Além de diagnóstico, este aparelho tem caráter terapêutico, levando a um aumento de 40 a 79\% no torque máximo do lado amputado e de 16 a 43\% no lado não amputado 7 .

A utilização desse recurso para a avaliação funcional torna mais relevante os parâmetros e objetivos para a reabilitação, configurando maior probabilidade da recuperação funcional destes pacientes, possibilitando manutenção e desenvolvimento da força muscular necessária para melhor controle e uso da prótese pelo paciente ${ }^{7}$.

Este trabalho tem por objetivo avaliar o desempenho muscular do quadril do membro amputado e do membro não amputado de adultos jovens com amputação transfemoral unilateral, e comparar o desempenho muscular do quadril entre o membro dominante e não dominante de adultos jovens amputados (transfemoral) e controles não amputados.

\section{MÉTODOS}

O estudo foi delineado como um estudo observacional transversal e aprovado pelo Comitê de Ética da Faculdade de Ciências da Saúde da Universidade de Brasília. Todos os participantes voluntários assinaram o Termo de Consentimento Livre e Esclarecido.

Participaram deste estudo quatro voluntários do gênero masculino. No centro de Treinamento de Educação Especial (CETEFE), Brasília, DF, Brasil, foram selecionados dois voluntários com amputação transfermoral, com idades de 30 e 29 anos, 66 e $70 \mathrm{Kg}$ de massa corporal e 1,75 e 1,82 metros de estatura corporal. Na própria universidade foram recrutados dois voluntários sem amputação de membros inferiores para compor o grupo controle, com idade de 20 e 25 anos, 76 e 76,3 Kg de massa corporal e 1,82 metros de estatura corporal.

A coleta de dados foi realizada no Laboratório de Análise de Marcha da Universidade de Brasília, Campus Ceilândia (FCE), em um ambiente tranquilo, com controle da temperatura $\left(24^{\circ} \mathrm{C}\right)$ e propício para as coletas. Inicialmente foi realizada a anamnese inicial, na qual foram colhidos dados pessoais do voluntário, características antropométricas, funcionais, tempo e frequência da prática regular de exercício físico. Em seguida, para avaliação do desempenho muscular isométrico dos flexores e extensores do quadril foi utilizado o dinamômetro isocinético Biodex System 4 Pro ${ }^{\circledR}$ (Biodex Medical Systems Inc., Shirley, NY, USA). O dinamômetro isocinético é um aparelho eletromecânico com sistema servomotor, que atualmente se apresenta todo computadorizado. Neste instrumento é possível quantificar valores de força muscular, resistência, potência, trabalho, torque e contrações musculares. Para realização do teste isométrico dos músculos flexores e extensores do quadril foi realizada calibração do equipamento conforme instruções do fabricante e observados os princípios do teste de desempenho muscular: educação do paciente, aquecimento prévio, posicionamento, estabilização do corpo do paciente e alinhamento do eixo articular e correção da gravidade antes de cada coleta para evitar intercorrências nos resultados. Os voluntários foram avaliados em decúbito dorsal (figuras 1 e 2) e o eixo rotatório do dinamômetro foi alinhado ao trocanter maior do fêmur, eixo dos movimentos de flexão e extensão do quadril. Durante a avaliação os voluntários foram estabilizados firmemente à cadeira do dinamômetro por meio de cintos na região do tronco, do quadril e da coxa contralateral à avaliação a fim de evitar compensações indesejáveis e foram orientados a segurar no aparelho para maior conforto. As medidas foram coletadas bilateralmente, em duas séries de contrações isométricas, nas angulações de 30 e 60 graus, com duração de 10 segundos. Cada série tinha um total de seis repetições, contabilizando três flexões e três extensões e um intervalo de 20 segundos para cada repetição. Foi utilizado intervalo de repouso de 120 segundos entre cada série para minimizar efeitos de fadiga muscular, foi dado encorajamento verbal padronizado usando palavras e frases de incentivo (força para cima, força para baixo, força) e foi utilizado feedback visual na tela do computador, na qual os voluntários podiam observar o gráfico da força que estava realizando durante o teste.

As análises estatísticas foram processadas utilizando-se o programa SPSS 16.0. As variáveis "média de pico de torque" e "pico de torque corrigido pela massa corporal" dos músculos flexores e extensores do

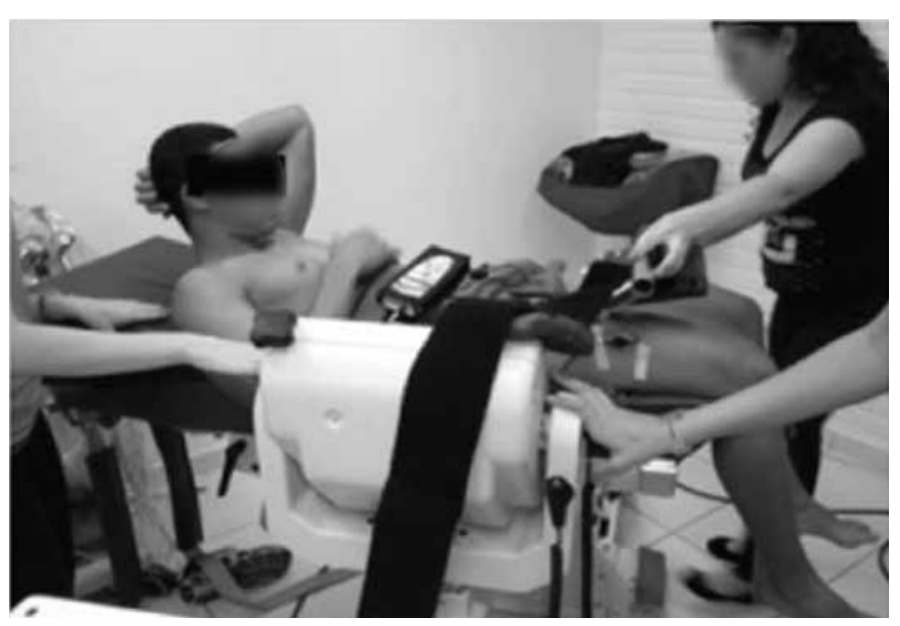

Figura 1. Voluntário não amputado posicionado no dinamômetro isocinético. 
quadril posicionado a 30 e 60 graus, foram analisadas de forma descritiva utilizando medidas de tendência central (média) e de variabilidade (desvio-padrão). Para avaliação dos resultados dos voluntários amputado e não amputados, o lado considerado dominante nos pacientes amputados foi o lado não amputado.

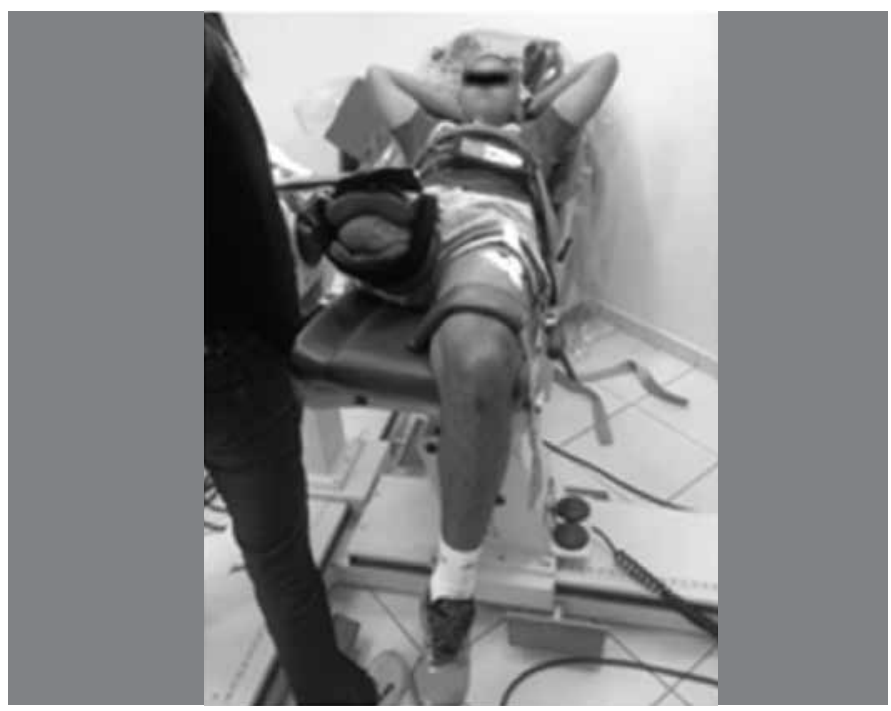

Figura 2. Voluntário amputado posicionado no dinamômetro isocinético.

\section{RESULTADOS}

Nessa amostra de quatro pacientes, esta pesquisa apresenta resultados de um estudo piloto no qual esses dados iniciais já podem confirmar algumas hipóteses e elaborar novas. Em um estudo com mais voluntários, será possível analisar mais variáveis e fazer comparações estatisticamente significativas.

Os dados demográficos referentes à idade, altura, peso corporal, índice de massa corporal (IMC) e exercícios físicos encontram-se na tabela 1. Os voluntários não eram tabagistas e nem etilistas.

Na tabela 2 estão apresentados os dados referentes ao desempenho muscular do paciente amputado, comparando-se as médias entre o lado amputado e o lado não amputado na angulação de 30 graus do dinamômetro isocinético.

Na tabela 3 encontram-se os dados referentes ao desempenho muscular do paciente amputado, comparando as médias entre o lado amputado e o lado não amputado na angulação de 60 graus. Observa-se que o membro amputado apresenta menor pico de torque, seja média ou valor corrigido pela massa corporal, em relação ao não amputado, tanto na flexão como extensão do quadril a 30 e a 60 graus.

Tabela 1. Média dos dados antropométricos.

\begin{tabular}{c|c|c}
\hline Variável & Amputados & Não amputados \\
\hline Idade $($ anos $)$ & $29,5 \pm 0,7$ & $22,5 \pm 3,5$ \\
\hline Altura $(\mathrm{m})$ & $1,78 \pm 0,049$ & $1,77 \pm 0,06$ \\
\hline Massa corporal $(\mathrm{kg})$ & $68 \pm 2,8$ & $76,15 \pm 0,2$ \\
\hline Imc $\left(\mathrm{kg} / \mathrm{m}^{2}\right)$ & $21,34 \pm 0,29$ & $24,21 \pm 1,66$ \\
\hline Exercícios - vezes por semana & $4,5 \pm 2,1$ & $5,5 \pm 2,1$ \\
\hline Exercícios - duração $(\mathrm{h})$ & $2,5 \pm 0,7$ & 2 \\
\hline
\end{tabular}

Tabela 2. Comparação entre o membro amputado e não amputado.

\begin{tabular}{c|c|c|c}
\hline \multicolumn{2}{c|}{ Variável } & MA & MNA \\
\hline \multirow{2}{*}{$\begin{array}{c}\text { Média de pico de } \\
\text { Torque }(\mathrm{nm})\end{array}$} & Flexores 30 graus & $68,2 \pm 26,44$ & $160,65 \pm 31,60$ \\
\cline { 2 - 4 } & Extensores 30 graus & $99,6 \pm 51,76$ & $188,7 \pm 38,89$ \\
\hline $\begin{array}{c}\text { Pico de torque por massa } \\
\text { corporal }(\mathrm{nm} / \mathrm{kg})\end{array}$ & Flexores 30 graus & $99,75 \pm 34,86$ & $235,95 \pm 36,55$ \\
\cline { 2 - 4 } & Extensores 30 graus & $145,8 \pm 69,29$ & $277,05 \pm 45,46$ \\
\hline
\end{tabular}

Na tabela 4 observam-se os dados médios da força muscular dos pacientes amputados e não amputados na angulação de 30 graus e na tabela 5 na angulação de 60 graus.

Tabela 3. Comparação entre o membro amputado e não amputado.

\begin{tabular}{c|c|c|c}
\hline \multicolumn{2}{c|}{ Variável } & MA & MNA \\
\hline \multirow{2}{*}{$\begin{array}{c}\text { Média de pico de } \\
\text { torque (Nm) }\end{array}$} & Flexores 60 graus & $55,3 \pm 17,3$ & $147 \pm 28,4$ \\
\cline { 2 - 4 } & Extensores 60 graus & $135,9 \pm 26,3$ & $176,6 \pm 23,2$ \\
\hline \multirow{2}{*}{$\begin{array}{c}\text { Pico de torque por massa } \\
\text { corporal (Nm/kg) }\end{array}$} & Flexores 60 graus & $81,0 \pm 21,9$ & $216,1 \pm 32,6$ \\
\cline { 2 - 4 } & Extensores 60 graus & $199,7 \pm 30,4$ & $259,7 \pm 23,2$ \\
\hline
\end{tabular}

* MA: membro amputado; MNA: membro não amputado.

Tabela 4. Comparação entre voluntários amputados e não amputados.

\begin{tabular}{|c|c|c|c|c|}
\hline Angulação & \multicolumn{2}{|c|}{ Variável } & VA & VNA \\
\hline \multirow{4}{*}{$\begin{array}{l}\text { Flexores } \\
30 \text { Graus }\end{array}$} & \multirow{2}{*}{$\begin{array}{l}\text { Média de pico de } \\
\text { torque }(\mathrm{nm})\end{array}$} & $\begin{array}{c}\text { Membro } \\
\text { dominante }\end{array}$ & $160,6 \pm 31,6$ & $154 \pm 23,19$ \\
\hline & & $\begin{array}{c}\text { Membro não } \\
\text { dominante }\end{array}$ & $68,2 \pm 26,4$ & $138,9 \pm 14,1$ \\
\hline & \multirow{2}{*}{$\begin{array}{c}\text { Pico de torque por } \\
\text { massa corporal } \\
(\mathrm{nm} / \mathrm{kg})\end{array}$} & $\begin{array}{c}\text { Membro } \\
\text { dominante }\end{array}$ & $235,9 \pm 36,5$ & $203 \pm 29,69$ \\
\hline & & $\begin{array}{c}\text { Membro não } \\
\text { dominante }\end{array}$ & $99,7 \pm 34,86$ & $182,6 \pm 17,8$ \\
\hline \multirow{4}{*}{$\begin{array}{c}\text { Extensores } \\
30 \text { Graus }\end{array}$} & \multirow{2}{*}{$\begin{array}{l}\text { Média de pico } \\
\text { de torque (nm) }\end{array}$} & $\begin{array}{c}\text { Membro } \\
\text { dominante }\end{array}$ & $188,7 \pm 38,89$ & $209,05 \pm 0,07$ \\
\hline & & $\begin{array}{c}\text { Membro não } \\
\text { dominante }\end{array}$ & $99,9 \pm 51,26$ & $110,8 \pm 17,6$ \\
\hline & \multirow{2}{*}{$\begin{array}{c}\text { Pico de torque por } \\
\text { massa corporal } \\
(\mathrm{nm} / \mathrm{kg})\end{array}$} & $\begin{array}{c}\text { Membro } \\
\text { dominante }\end{array}$ & $277 \pm 45,4$ & $275,9 \pm 1,06$ \\
\hline & & $\begin{array}{c}\text { Membro não } \\
\text { dominante }\end{array}$ & $145,8 \pm 69,29$ & $145,7 \pm 22,5$ \\
\hline
\end{tabular}

*VA: voluntário amputado; VNA: voluntário não amputado.

Tabela 5. Comparação entre voluntários amputados e não amputados.

\begin{tabular}{|c|c|c|c|c|}
\hline $\begin{array}{l}\text { Angulação } \\
\text { isocinético }\end{array}$ & \multicolumn{2}{|c|}{ Variável } & VA & VNA \\
\hline \multirow{4}{*}{$\begin{array}{c}\text { Flexores } 60 \\
\text { graus }\end{array}$} & \multirow{2}{*}{$\begin{array}{c}\text { Média de pico de } \\
\text { torque }(\mathrm{nm})\end{array}$} & $\begin{array}{c}\text { Membro } \\
\text { dominante }\end{array}$ & $147,1 \pm 28,42$ & $147,95 \pm 30,9$ \\
\hline & & $\begin{array}{c}\text { Membro não } \\
\text { dominante }\end{array}$ & $55,35 \pm 17,32$ & $140,75 \pm 5,02$ \\
\hline & \multirow{2}{*}{$\begin{array}{c}\text { Pico de torque por } \\
\text { massa corporal } \\
(\mathrm{nm} / \mathrm{kg})\end{array}$} & $\begin{array}{c}\text { Membro } \\
\text { dominante }\end{array}$ & $216,1 \pm 32,66$ & $194,5 \pm 39,88$ \\
\hline & & $\begin{array}{c}\text { Membro não } \\
\text { dominante }\end{array}$ & $81,5 \pm 21,99$ & $185,1 \pm 5,79$ \\
\hline \multirow{4}{*}{$\begin{array}{c}\text { Extensores } 60 \\
\text { graus }\end{array}$} & \multirow{2}{*}{$\begin{array}{l}\text { Média de pico de } \\
\text { torque }(\mathrm{nm})\end{array}$} & $\begin{array}{c}\text { Membro } \\
\text { dominante }\end{array}$ & $179,5 \pm 19,23$ & $261,4 \pm 37,33$ \\
\hline & & $\begin{array}{c}\text { Membro não } \\
\text { dominante }\end{array}$ & $157,4 \pm 3,95$ & $152,15 \pm 8,27$ \\
\hline & \multirow{2}{*}{$\begin{array}{c}\text { Pico de torque por } \\
\text { massa corporal } \\
(\mathrm{nm} / \mathrm{kg})\end{array}$} & $\begin{array}{c}\text { Membro } \\
\text { dominante }\end{array}$ & $259,7 \pm 23,26$ & $343,85 \pm 50,5$ \\
\hline & & $\begin{array}{l}\text { Membro não } \\
\text { dominante }\end{array}$ & $199,7 \pm 30,4$ & $200,1 \pm 11,73$ \\
\hline
\end{tabular}

*VA: voluntário amputado; VNA: voluntário não amputado.

\section{DISCUSSÃO}

O indivíduo que vive com a perda de um membro em nível transfemoral, enfrenta diversos desafios, tais como: exigências para aumentar a energia, problemas de equilíbrio e estabilidade. Nas amputações acima do joelho e nas desarticulações do mesmo, é perdida a flexo-extensão do joelho que regula o comprometimento do membro inferior tornando 
e a oscilação do centro de gravidade suavizando a caminhada ${ }^{8}$. Desta forma, a perda de uma parte da perna demanda ajustes musculares para a manutenção da postura de pé e a sua locomoção.

Durante a marcha na fase de balanço, o amputado deve iniciar o balanço com flexão do quadril para depois realizar a flexão do joelho mecânico e levar o joelho em extensão se preparando para o contato inicial. Nesta fase, pode-se encontrar vários desvios como a hiperlordose, compensação funcional dos amputados transfemorais proximais. O impacto no final da extensão, observado no plano sagital durante a fase final do balanço, pode ser audível e geralmente está relacionada com os ajustes do joelho mecânico causados por uma insuficiente resistência a extensão ou por um movimento brusco da flexão do quadril na fase inicial do impulso e dos extensores do quadril na fase final do balanço?

Analisando os dados é possível observar uma diferença clinicamente importante, ainda não calculada estatisticamente, entre os membros amputado e não amputado. É visível que o membro amputado apresenta valores, visto que a amputação gera uma fraqueza nos músculos residuais do quadril, e este é um fator contribuinte para anormalidades na marcha de pacientes com amputação transfemoral ${ }^{10}$.

Como mecanismo compensatório da marcha, espera-se maior potência e trabalho das estruturas do quadril proporcional da perna intacta ${ }^{10,11}$. Observa-se também uma média de pico de torque mais elevada no grupo muscular de extensores quando comparado aos flexores. Os músculos extensores do quadril de amputados podem apresentar mecanismos de adaptação muscular no lado protético, pois os extensores do quadril devem fornecer mais poder para facilitar a propulsão e manter os parâmetros de marcha como velocidade, comprimento do passo, tempo, postura e cadência ${ }^{5}$.

São escassos na literatura estudos sobre valores de força muscular de quadril em amputados transfemoral. As atividades diárias como caminhar, sentar e levantar de uma cadeira ou subir e descer escadas necessitam da força de diferentes grupos musculares, especialmente no caso de amputados transfemorais. Nas tabelas 4 e 5 é possível identificar a diferença em relação à média de pico de torque para o lado dominante e o não dominante dos pacientes amputados e não amputados. Os valores médios de pico de torque do membro não dominante dos pacientes amputados é visivelmente menor do que a média de pico de torque do membro não dominante dos pacientes não amputados, resultado já esperado considerando a amputação transfemoral. Identificando-se os componentes biomecânicos envolvidos nas atividades diárias dos amputados, pretende-se construir e otimizar uma prótese de membro inferior que viabilize um padrão de marcha e de atividades mais próximas da normalidade e com menor desgaste articular.

\section{CONCLUSÃO}

A amputação transfemoral de quadril provoca importantes deficiências de estrutura e função corporal no membro amputado, como a observada redução da força muscular, que pode determinar comprometimentos funcionais, limitações de atividade e mobilidade. Este trabalho é um ponto de partida para futuras investigações da associação do desempenho muscular e capacidade funcional nestes pacientes visando o esclarecimento do impacto funcional da amputação e a determinação do efeito de intervenções diretas (treinamentos funcionais) ou indiretas (tecnologias assistivas) na melhora do desempenho funcional desses pacientes.

\section{AGRADECIMENTOS}

Agradecemos ao CETEFE por possibilitar o contato com os voluntários amputados e ao apoio financeiro do CNPq, do ProlC/UnB e do Decanto de Pós-Graduação (DPP) da UnB.

$\overline{\text { Todos os autores declararam não haver qualquer potencial conflito de }}$ interesses referente a este artigo.

\section{REFERÊNCIAS}

1. Oliveira M, Vilagra JM. Independência funcional e satisfação em pacientes com amputação transfemoral [anais]. In: III Congresso Internacional de Saúde da UEM, Maringá, 2009.

2. Kisner C, Colby LA. Exercícios terapêuticos: fundamentos e técnicas. 4ed. São Paulo: Manole; 2005

3. Zabka FF, Valente HG, Pacheco AM. Avaliação isocinética dos músculos extensores e flexores de joelho em jogadores de futebol profissional. Rev Bras Med Esporte. 2011;17(3):189-92.

4. Silva Neto M, Simoes R, Grangeiro Neto JA, Cardone CP. Avaliação isocinética da força muscular em atletas profissionais de futebol feminino. Rev Bras Med Esporte. 2010;16(1):33-5.

5. Croisier JL, Maertens De Noordhout B, Maquet D, Camus G, Hac S, Feron F, et al. Isokinetic evaluation of hip strength muscle groups in unilateral lower limb amputees. Isokinet Exerc Sci. 2001;9(4):163-9.

6. Terreri ASA, Greve JMD, Amatuzzi MM. Avaliação isocinética no joelho do atleta", Rev Bras Med Esporte. 2001;7(2):62-6.
7. Tanaka AP. Avaliação fisioterápica do paciente com amputação. In: Pedrinelli A. Tratamento do paciente com amputação. São Paulo: Rocca; 2004. p. 165-72.

8. Carvalho JA. Amputações de membros inferiores: em busca da plena reabilitação. $2^{2}$ ed. São Paulo: Manole; 2003.

9. Wilken JM, Marin R. Gait analysis and training of people with limb loss. In: Care o the Combat Amputee; 2009. p. 535-52.

10. Vallery H, Burgkart R, Hartmann C, Mitternacht J, Riener R, Buss M. Complementary limb motion estimation for the control of active knee prostheses. Biomed Tech (Berl). 2011;56(1):45-51.

11. Silverman AK, Fey NP, Portillo A, Walden JG, Bosker G, Neptune RR. Compensatory mechanisms in below-knee amputee gait in response to increasing steady-state walking speeds. Gait Posture. 2008;28(4):602-9. 